\title{
Perspective
}

\section{Open access spreadsheet application for learning spontaneous breathing mechanics and mechanical ventilation}

\section{Introduction}

Mechanical ventilation, either invasive or noninvasive, is crucial to treat patients with acute or chronic respiratory failure in intensive care units and hospital wards. Optimal gas exchange is not easy to achieve in patients with respiratory failure since a considerable number of variables and mechanisms involving several organs and systems play a substantial role. Moreover, an added difficulty when managing invasive mechanical ventilation is that improvement of gas exchange must be achieved by minimising the risk of ventilatorinduced lung injury. Hence, optimal application of mechanical ventilation requires fine tuning of the ventilator settings, tailoring them to each patient's needs. This process cannot be carried out by trial and error but based on a solid knowledge of the concepts and physical laws governing respiratory mechanics. Accordingly, it is important that medical students achieve a good background understanding of respiratory mechanics in undergraduate courses of physiology, and that this training is refreshed later when the student is introduced to mechanical ventilation learning and further when starting clinical training in this therapy [1].

Given the lack of simple, adaptable, and freely available tools for training in the basic concepts of respiratory mechanics and mechanical ventilation, based on the widely used Microsoft Excel spreadsheet platform, we designed a practical tool for medical students covering the basic concepts of respiratory mechanics and their application to mechanical ventilation. Herein, we briefly describe this innovative teaching tool and provide both teacher and student versions of the spreadsheets as open access supplementary files. In the student version some data and worksheets for the spreadsheets are hidden, and workbook structure is protected (a password can be included) except the content of the cells to be changed by the students.

\section{Description of the tool}

\section{Part 1: mechanics of the spontaneous breathing cycle}

The aim of this practical exercise is to help the student to understand the basis of spontaneous breathing cycle mechanics at rest. It is based on numerical simulation and shows, numerically and graphically, how the elastic and resistive properties of the lungs and rib cage determine the mechanics of the respiratory cycle. The simulation is based on a flow signal, which was recorded with a pneumotachograph in a resting healthy subject breathing spontaneously. A first Introduction worksheet presents the description of the application and poses the questions that the student should answer when proceeding through the exercise. As illustrated in figure 1, worksheets DATA 1 and FIG 2 depict typical baseline

@ERSpublications

Description and presentation of an open access spreadsheet application for learning spontaneous breathing mechanics and mechanical ventilation https://bit.ly/2TyXo1C

Cite as: Navajas D Almendros I, Otero J, et al Open access spreadsheet application for learning spontaneous breathing mechanics and mechanical ventilation. Breathe 2021; 17: 210012.

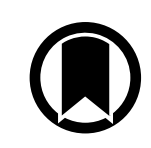

CrossMark 
a)

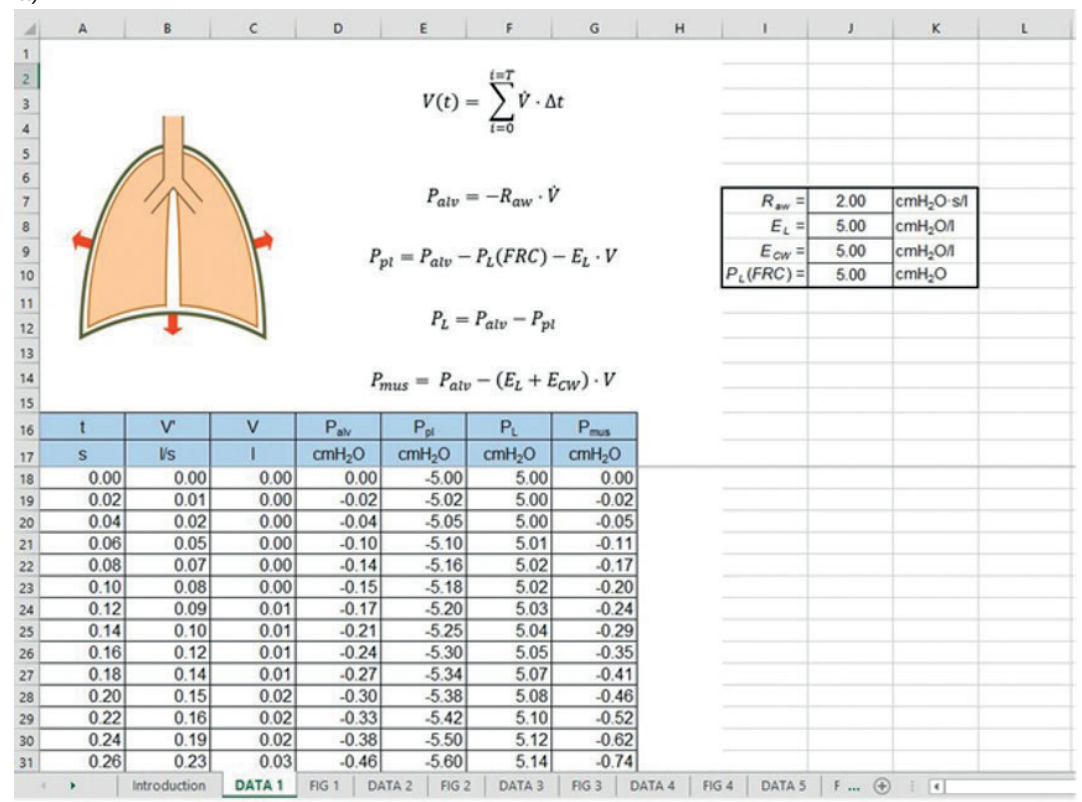

b)

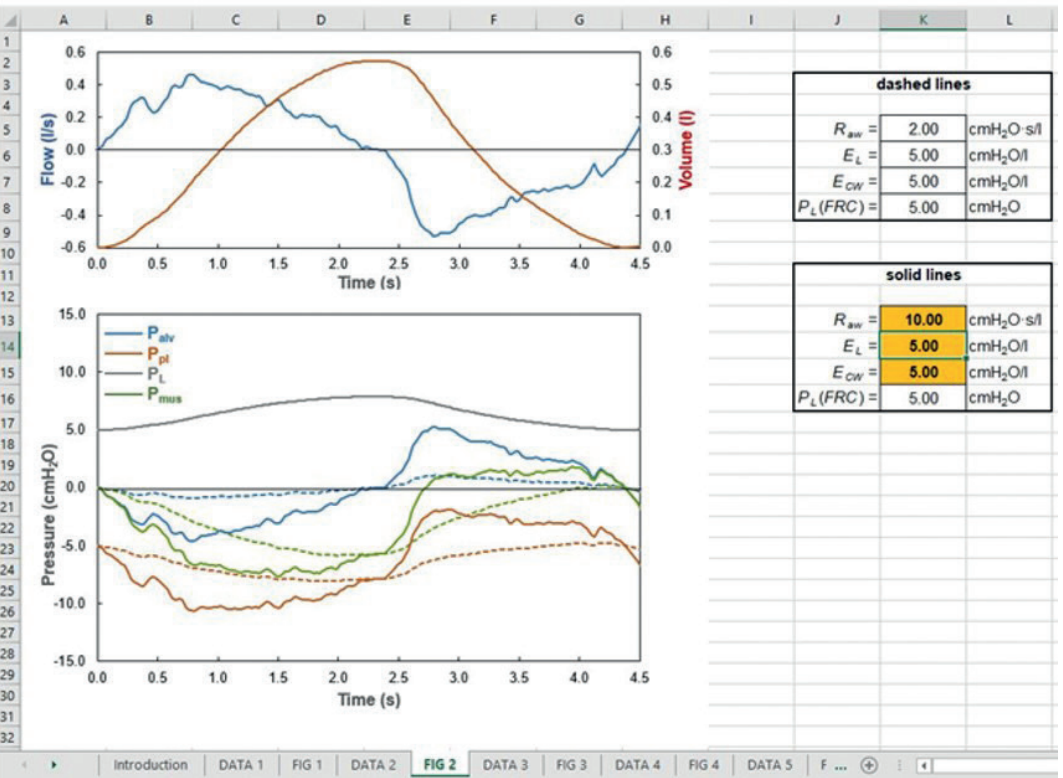

Figure 1 Mechanics of the spontaneous breathing cycle (Part 1). a) Screenshot of tab DATA 1 showing baseline values of airway resistance $\left(R_{\text {aw }}\right)$, lung elastance $\left(E_{L}\right)$, and chest wall elastance $\left(E_{C W}\right)$, the values of the flow signals: flow $(\dot{V})$, volume $(V)$, and alveolar $\left(P_{a l v}\right)$, pleural $\left(P_{p}\right)$, transpulmonary $\left(P_{L}\right)$ and muscular $\left(P_{\text {mus }}\right)$ pressures. b) Screenshot of tab FIG 2 showing the time course of the signals for baseline parameters (dashed lines) and when $R_{\text {aw }}$ was increased from $2 \mathrm{cmH}_{2} \mathrm{O} \cdot \mathrm{s} \cdot \mathrm{L}^{-1}$ to $10 \mathrm{cmH} \mathrm{CH}_{2} \mathrm{O} \cdot \mathrm{s} \cdot \mathrm{L}^{-1}$ (solid lines). Orange cells allow students to change of their numerical values. See text for explanation.

values of airway resistance $\left(R_{\mathrm{aw}}=2 \mathrm{cmH} \mathrm{CH}_{2} \mathrm{O} \cdot \mathrm{s} \cdot \mathrm{L}^{-1}\right)$, lung elastance $\left(E_{\mathrm{L}}=5 \mathrm{cmH}_{2} \mathrm{O} \cdot \mathrm{L}^{-1}\right)$, and chest wall elastance $\left(E_{\mathrm{CW}}=5 \mathrm{cmH}_{2} \mathrm{O} \cdot \mathrm{L}^{-1}\right)$, the values of the flow signal $(\dot{V})$ and the corresponding volume $(V)$ computed by $\dot{V}$ integration. It also presents the time course of alveolar $\left(P_{\mathrm{alv}}=-R_{a w} \cdot \dot{V}\right)$, pleural $\left(P_{\mathrm{pl}}=P_{\mathrm{alv}}{ }^{-}\right.$ $\left.P_{L}(\mathrm{FRC})-E_{L} \cdot V\right)$, transpulmonary $\left(P_{L}=P_{\text {alv }}-P_{p l}\right)$ and muscular $\left(P_{\mathrm{mus}}=P_{\mathrm{alv}}-\left(E_{\mathrm{L}}+E_{\mathrm{CW}}\right) \cdot V\right.$ pressures required to induce the specific ventilation $(\dot{V}, V)$ for the set values of $R_{a w}, E_{L}$ and $E_{\mathrm{CW}}$, by considering that the functional residual capacity (FRC) of the subject is
$2.5 \mathrm{~L}$ and that the value of $P_{L}$ at this volume is $P_{L}$ $(\mathrm{FRC})=5 \mathrm{cmH}_{2} \mathrm{O}$. The student can see the numerical values of the signals at any time in the table in tab DATA 1 or in FIG 1 by placing the tip of the mouse pointer over the corresponding point of the graph. As can be seen in the Introduction worksheet, the student is asked to go into the details of the data and figures to understand the time course of the different signals and their relationship. For instance, to calculate the duration of inspiration and expiration, and the values of respiratory rate, tidal volume and minute ventilation. Also, to analyse whether each of the pressures $\left(P_{\mathrm{alv}}, P_{\mathrm{pl}}, P_{\mathrm{L}}\right.$ and $\left.P_{\text {mus }}\right)$ are positive, zero or negative at different times in inspiration and expiration and whether one of the pressures is more positive/negative than another one. Finally, the student is asked to select the signal values at specific points (e.g. beginning/end of inspiration/expiration or points with equal flow/ volume) to derive $R_{\mathrm{aw}}, E_{\mathrm{L}}$ and $E_{\mathrm{CW}}$.

FIG 2 allows the student to freely modify the value of $R_{\mathrm{aw}}, \mathrm{E}_{\mathrm{L}}, \mathrm{E}_{\mathrm{CW}}$ and $P_{\mathrm{L}}(\mathrm{FRC})$. This is intended to challenge them to predict how the different pressure signals will change and then to confirm their prediction by comparing the new pressure signals with baseline ones (figure 1b). The student can modify only one respiratory parameter (for instance to model airway obstruction, lung or chest wall restrictions) to observe the specific change induced in each of the pressures, and they can also modify simultaneously several parameter values to observe the combined effect.

Subsequently, tabs FIG 3 to FIG 5 are aimed at challenging the student to determine the values of respiratory parameters from observation of the signals, therefore moving from deductive to inductive reasoning. In the corresponding graphical representations, the different pressures computed after one (or more) parameters has been modified (solid lines) are compared with those of the baseline healthy subject (dashed lines) of tab FIG 2 , and the student is asked which of the parameter(s) has been modified in each case.

\section{Part 2: mechanical ventilation}

The aim of this practical exercise is to understand the basics of mechanical ventilation by modelling the signals of $\dot{V}, V$, airway opening pressure $\left(P_{\text {ao }}\right)$ and alveolar pressure $\left(P_{\text {alv }}\right)$ during mechanical ventilation with tracheal intubation of a patient with an anatomical dead space of $150 \mathrm{~mL}$, alveolar dead space of $7 \%$, and with the respiratory musculature paralysed and the ventilator set to provide constant inspiratory flow. The following parameter values are taken as the baseline: minute ventilation $=7 \mathrm{~L} \cdot \mathrm{min}^{-1}$, respiratory rate $=10$ breaths per $\mathrm{min}$, inspiratory time $=2 \mathrm{~s}$, equipment dead space $=100 \mathrm{~mL}$ and positive end-expiratory pressure (PEEP) $=5 \mathrm{cmH}_{2} \mathrm{O}$. The endotracheal tube has a nonlinear resistance $R_{\mathrm{et}}=K_{1} \cdot \dot{V}+K_{2} \cdot \dot{V}^{2}$, with $K_{1}=1 \mathrm{cmH}_{2} \mathrm{O} \cdot \mathrm{s} \cdot \mathrm{L}^{-1}$ and $K_{2}=5 \mathrm{cmH}_{2} \mathrm{O} \cdot\left(\mathrm{s} \cdot \mathrm{L}^{-1}\right)^{2}$. An Introduction tab describes 
the application and poses the questions to be answered by the student. The first questions are to determine the values of tidal volume, ratio of inspiratory/expiratory time and alveolar ventilation.

Tab FIG 1 simulates $\dot{V}, V, P_{\text {alv }}$ and $P_{\text {ao }}$ signals in a mechanically ventilated patient without respiratory disease subjected to surgery, with the same baseline values as in Part 1. The student is asked to describe the specific time profile of the signals and to explore their values by placing the tip of the mouse pointer over the point of interest. Tab FIG 2 allows the student to assess the effect of the different parameters in the signals, by first predicting the effects of an up to three-fold increase in $R_{\mathrm{aw}}, E_{\mathrm{L}}$, and PEEP and subsequently checking their prediction by changing the parameter values in the spreadsheet (figure $2 \mathrm{a}$ ). The student is also asked to assess the effect of the endotracheal tube by setting $K_{1}=0$ and $K_{2}=0$.

Tabs FIG 3 to FIG 5 simulate mechanical ventilation in patients with single or combined changes in $R_{\mathrm{aw}}, E_{\mathrm{L}}$, and PEEP and compare the ventilation signals with the baseline ones (tab FIG 1). The student is asked to analyse the changes that occurred during both inspiration and expiration and to answer which of the parameter(s) have been altered in each case. Finally, tabs FIG 6 and DATA 6 simulate the measurement of the patient's mechanics by an end-inspiratory airway occlusion manoeuvre (figure $2 b$ ). The student is asked to describe and interpret the time course of signals routinely displayed by ventilators $(\dot{V}$, $\left.V, P_{\text {ao }}\right)$ along the manoeuvre and to compute $R_{\text {aw }}$ and the total elastance of the respiratory system $\left(E_{\mathrm{RS}}=E_{\mathrm{L}}+E_{\mathrm{CW}}\right)$ before and after correction for the endotracheal tube.

\section{Discussion}

Acquiring competence in treatments using complex medical devices, such as mechanical ventilators, requires a good theoretical and practical background [2]. The practitioner should understand the mechanical concepts and equations relating the different variables involved in ventilation in order to succeed in setting an effective and safe combination of parameters for each patient condition. There are available educational tools to improve the training of physicians in mechanical ventilation based on patient simulators or manikins [1, 3-6], allowing the student to observe how varying the patient's mechanics or the ventilator settings impacts on the $\dot{V}, V$, and $P_{a o}$ signals recorded by the ventilator. However, to the best of our knowledge, an educational tool like the one we present here going into detail to better understand the problem and to interpret and predict the values of the variables involved in spontaneous breathing and mechanical ventilation monitoring is not easily available.

The described practical exercises are aimed at improving understanding of the most basic a)
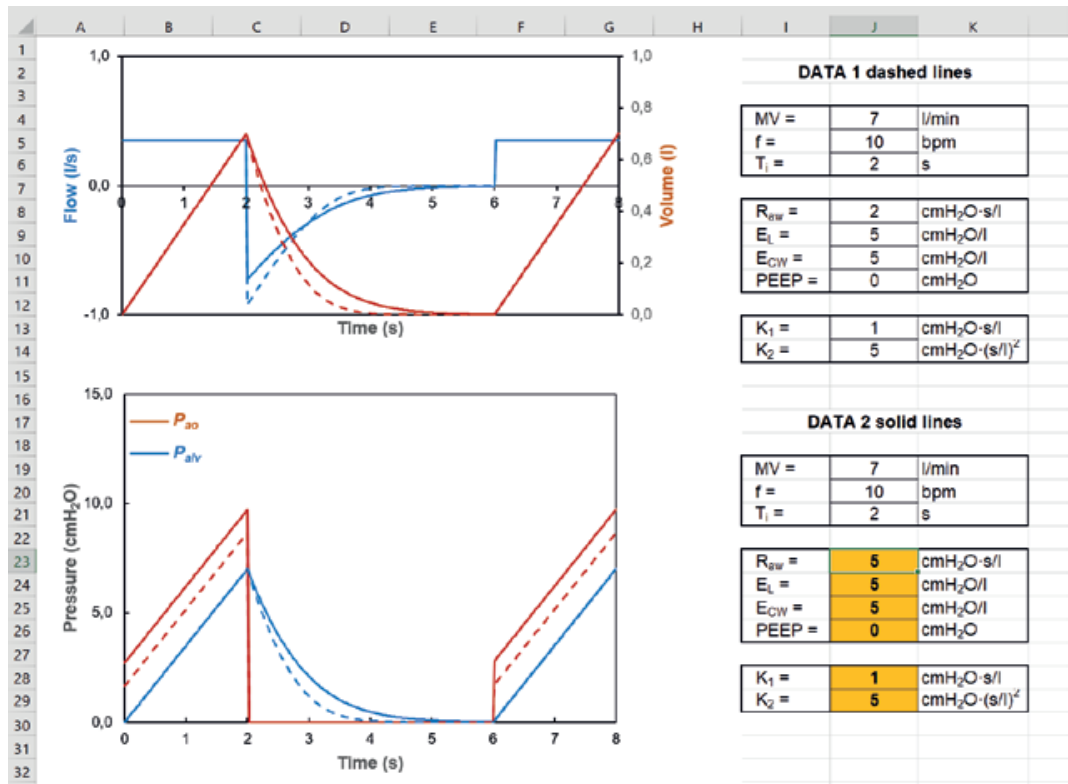

DATA 2 solid lines

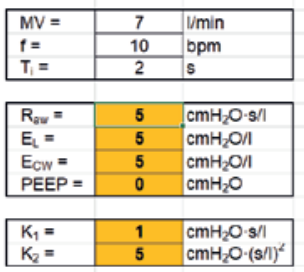

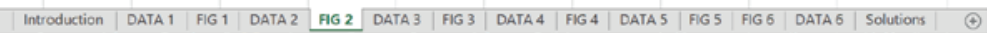
b)

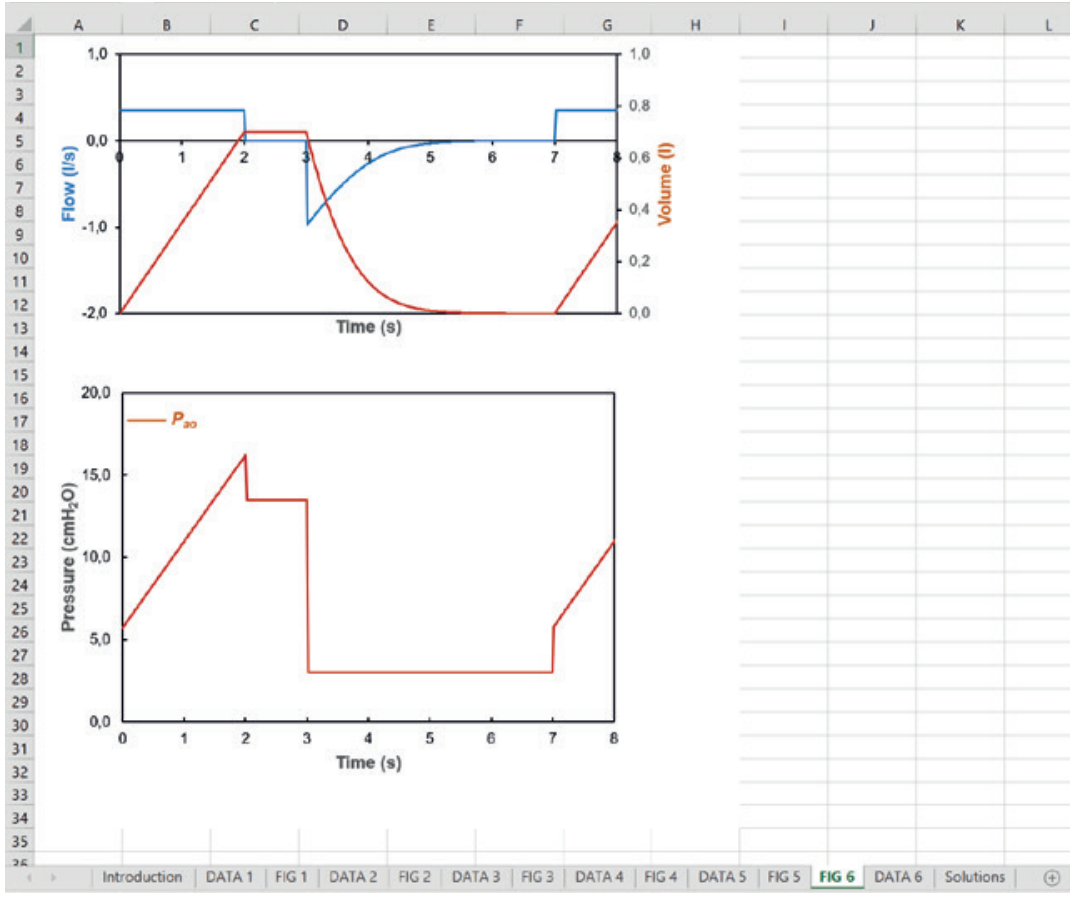

Figure 2 Mechanical ventilation (Part 2). a) Screenshot of tab FIG 2 showing the simulated signals offlow, volume and airway opening $\left(P_{a 0}\right)$ and alveolar $\left(P_{\text {alv }}\right)$ pressures during mechanical ventilation. Dashed and solid lines correspond to the baseline respiratory parameters and when respiratory resistance $\left(R_{\text {aw }}\right)$ was increased from $2 \mathrm{cmH}_{2} \mathrm{O} \cdot \mathrm{s} \cdot \mathrm{L}^{-1}$ to $5 \mathrm{cmH}_{2} \mathrm{O} \cdot \mathrm{s} \cdot \mathrm{L}^{-1}$, respectively. b) Screenshot of tab FIG 6 showing the simulated signals of flow, volume and $P_{\text {ao }}$ provided by $a$ conventional ventilator during an end-expiratory occlusion manoeuvre to monitor the patient's respiratory mechanics. Orange cells allow students to change of their numerical values. MV: minute ventilation; f: respiratory rate; $T_{i}$ : inspiratory time. See text for explanation.

concepts of respiratory mechanics, both in spontaneous breathing at rest and during mechanical ventilation. To this end, we model the respiratory system by simple linear resistances and 
elastances, and we illustrate the changes elicited by obstructive/restrictive conditions by simply changing the values of resistance and elastance, while retaining the typical breathing pattern of a healthy subject at rest. We took this simplified approach to avoid using nonlinear equations that account for flow-dependent airway resistance or volume-dependent lung/chest wall elastances. Furthermore, for the sake of simplicity, we do not implement mechanically complex models such as those accounting for expiratory flow limitation or multicompartmental respiratory systems [7-9]. However, these model refinements could be implemented if required by the learning objectives.

The educational tool presented herein has several advantages from an applicability viewpoint. Indeed, in addition to a completely open teacher version, we provide a student version in which all parts that not do not require student modification are blocked (by the Excel tools) to ensure full robustness. Moreover, the fact that our open access tool is based on what is the probably most widely available spreadsheet application allows teachers and students use it in a simple way with no additional cost. Our approach could be easily implemented into an open-software spreadsheet platform in cases where students do not have access to Excel. Finally, owing to the fact that the spreadsheet implementation does not require special skills in computer coding, as mentioned above, the details of the exercises can be easily changed for potential adaptation to the specific needs of an ample variety of students, from the most novice ones to physicians who are ready to start their clinical practice on lung function and on mechanical ventilation.

\section{Affiliations}

Daniel Navajas, Isaac Almendros, Jorge Otero, Ramon Farré

Unitat de Biofísica i Bioenginyeria, Facultat de Medicina i Ciències de la Salut, Universitat de Barcelona, Barcelona, Spain.

This article has supplementary material available from breathe.ersjournals.com

\section{Conflict of interest}

None declared.

\section{References}

1. Keller JM, Claar D, Ferreira JC, et al. Mechanical ventilation training during graduate medical education: perspectives and review of the literature. J Grad Med Educ 2019; 11: 389-401.

2. Dellaca RL, Veneroni C, Farré R. Trends in mechanical ventilation: are we ventilating our patients in the best possible way? Breathe (Sheff) 2017; 13: 84-98.

3. Spadaro S, Karbing DS, Fogagnolo A, et al. Simulation training for residents focused on mechanical ventilation: a randomized trial using mannequin-based versus computer-based simulation. Simul Healthc 2017; 12: 349-355.

4. Acho M, Lee AC, Lee BW. Ventilators for nonintensivists. Basic modes and breath types. ATS Scholar 2020; 1: 195-196.

5. Acho M, Lee AC, Lee BW. Ventilators for nonintensivists. Reasonable initial ventilator settings for patients with acute respiratory distress syndrome. ATS Sch 2020; 1 : 197-198.

6. Acho M, Lee AC, Lee BW. Ventilators for nonintensivists: troubleshooting elevations in plateau pressure. ATS Sch 2020; 1: 498-499.

7. Bates JH, Rossi A, Milic-Emili J. Analysis of the behavior of the respiratory system with constant inspiratory flow. J Appl Physiol 1985; 58: 1840-1848.

8. Similowski T, Bates JH. Two-compartment modelling of respiratory system mechanics at low frequencies: gas redistribution or tissue rheology? Eur Respir J 1991; 4: 353-358.

9. Peslin R, Rotger M, Farre R, et al. Assessment of respiratory pressure-volume nonlinearity in rabbits during mechanical ventilation. J Appl Physiol 1996; 80: 1637-1648. 